\title{
Malaria among the pastoral communities of the Ngorongoro Crater Area, northern Tanzania
}

\author{
L.E.G. MBOERA ${ }^{*}$, R.C. MALIMA ${ }^{2}$, P.E. MANGESHO², K.P. SENKORO ${ }^{1}$ \& V. MWINGIRA ${ }^{2}$ \\ ${ }^{1}$ National Institute for Medical Research, P.O. Box 9653, Dar es Salaam, Tanzania, ${ }^{2}$ National Institute for Medical \\ Research, Ubwari Research Station, P.O. Box 81, Muheza, Tanzania
}

\begin{abstract}
This study was undertaken to investigate the malaria burden and epidemic predisposing factors in the villages within the Ngorongoro Conservation Area Authority. The study involved four villages namely, Osinon (1720m), Olbalbal $(1780 \mathrm{~m})$, Endulen $(1800 \mathrm{~m})$ and Oloirobi $(2100 \mathrm{~m})$ and was carried out in August 2004. High malaria parasite rates were observed among people living in relatively low altitudes than in the higher altitudes (Osinoni $=25.9 \%$, Endulen $=13.9 \%$, Olbalbal $=9.8 \%$ and Oloirobi $=3.6 \%$ ). On average all underfives in the study villages were having a fairly good $\mathrm{Hb}$ levels ranging between 8.7 to $10.6 \mathrm{~g} / \mathrm{dl}$. Very few individuals were having enlarged spleens in all study villages (Olbalbal $0.4 \%$, Oloirobi $0.7 \%$, Endulen $0.5 \%$, and Osinoni $1.6 \%$ ). No malaria transmitting mosquitoes were collected in the area. Health facilities were the best source of care for malaria. The majority (59.7\%) of the respondents were living over $4 \mathrm{~km}$ from the nearest health facility. Over $76 \%$ of the respondents had at least a member of their household who had suffered from malaria during the preceding year. The majority of the people in Ngorongoro knew the symptoms and mode of transmission of malaria. Poverty, ignorance, ineffectiveness of drugs, poor sanitation, scarcity of water and culture and traditions were considered as the major disease predisposing factors among the community of Ngorongoro. Despite frequent malaria epidemics, the communities in Ngorongoro had poor knowledge on appropriate means of malaria prevention and control. The frequent malaria epidemics in Ngorongoro are likely to be associated with population movements, community poor knowledge on transmission and control and changes in climate. There is need to strengthen community health education among the people of Ngorongoro. Moreover, an early warning systems need to be established among all health facilities to monitor any impending epidemic in future.
\end{abstract}

Key words: malaria, pastoral community, epidemic, highlands, Tanzania

\section{Introduction}

Malaria is the primary cause of ill health in Africa South of Sahara, causing an enormous health and economic burden. The World Health Organization (WHO) has estimated that malaria causes $270-450$ million illness episodes and 0.9-1.8 million deaths annually in Africa South of Sahara, the great majority in children under the age of 5 years (WHO, 1997). Tanzania's geographical profile provides a wide range of heterogeneity in the malaria transmission patterns and vectorial systems. Some areas have perennial and intense malaria transmission while others have intense but seasonal transmission. Yet other areas experience moderate transmission (Clyde, 1967) and sometimes epidemics (Mboera \& Kitua, 2001). Tanzania is probably among the worst hit malaria endemic country in Africa having areas with highest transmission, with over 400 infective bites per person per year (Ellman et al., 1998). There are very few areas where transmission is very low or absent.

Malaria is endemic throughout virtually all of Tanzania and accounts for over $30 \%$ of the national disease burden and is a threat to every one of the estimated 32 million (94\% of the population) living in areas with stable malaria transmission. Yearly incidence is

Correspondence: L.E.G. Mboera; Email: Imboera@nimr.or.tz estimated to be between 14 and 18 million cases. In addition, malaria is responsible for 100,000 to 125,000 deaths per year, with 70,000 to 80,000 of these deaths occurring in children aged $<5$ years.

Malaria is the leading cause of outpatient attendance for children aged $<5$ years $(38 \%)$, as well as for the $\geq 5$ year age group ( $32 \%$ ). It is the first ranked cause of death in hospitalised patients of all ages and the leading cause of admissions for children aged $<5$ years $(43 \%)$. The most vulnerable population groups are children aged $<5$ years $(7.1$ million) and pregnant women ( 1.69 million). The socio-economic impact of this disease is substantial, from costs associated with prevention and treatment, costs associated with deaths, and reduced workforce productivity and school absenteeism.

The Tanzania National Malaria Medium Term Strategic Plan 2002-2007 (NMMTSP) aims to reduce malaria mortality and morbidity in all 21 regions by $25 \%$ by 2007 and by $50 \%$ by 2010 . Four key strategic approaches are employed to reach this goal: (1) improved case management, (2) vector control through a country-wide use of insecticide-treated nets, (3) control of malaria in pregnancy, and (4) malaria epidemic prevention and control. However, several constraints to control have been identified to include antimalarial drug resistance, availability of 
antimalarial drugs, affordability of insecticide-treated bednets, human behaviour, constant population movements as well as ecological changes.

The highlands of Ngorongoro were known to be free from malaria until mid-1990s. Several malaria epidemics have since been reported. In 1994-95 malaria epidemics in the district were reported in highland areas of Ngarwa, Loliondo and Sakala (1000$3000 \mathrm{~m})$. In 1995 alone, malaria accounted for $57.2 \%$ and $71.2 \%$ of all hospital outpatient and in-patient attendance respectively (Alilio et al., 1995). Recently, malaria outbreaks have been reported in villages of Endulen, Osinon and Olbalbal in the Ngorongoro Division, which form part of the Ngorongoro Conservation Area Authority. From 1998 to 1999, there was an increase in the number malaria by $193 \%$ in Endulen and $120 \%$ in Olbalbal. The increase in malaria cases was markedly high in children under 5 years of age. These increases were always observed during the rainy season. To date, malaria is number one public health problem in the district. Other important diseases include acute respiratory infections, pneumonia, eye infections and diarrhoea. The disease is seasonal, with most of the cases occurring during the months of January-March.

It has been reported that human migration is responsible for the malaria epidemics in the district (Alilio et al., 1995). Population migration in Ngorongoro district is common among the pastoralists. Such movements are usually initiated mainly by three factors. Firstly, tribal conflicts and fighting between the Maasai and minority tribes force people to flee their villages in fear of being attacked. Secondly, movements caused by other epidemics, particularly meningitis and dysentery have caused communities in some villages to run away from their homes. Thirdly, search for green pastures entails movements with animals to areas around lakes and big rivers within and outside the district during the dry season.

In order to produce evidence-based recommendations on appropriate and effective malaria intervention methods, research to determine the current malaria burden and epidemic predisposing factors was proposed to be undertaken in areas under the Ngorongoro Conservation Area Authority (NCAA). The main objective was to determine malaria disease burden and the dynamics of transmission in epidemic-prone areas of Ngorongoro Conservation Area Authority in Ngorongoro District in northern Tanzania.

\section{Materials and Methods}

\section{Study Site}

The study was conducted in August 2004 in an area under Ngorongoro Conservation Area Authority in Ngorongoro District ( $3^{\circ} 00^{\prime} \mathrm{S}, 35^{\circ} 15^{\prime} \mathrm{E}$ ). Ngorongoro district is characterised by highlands and mountains, particularly in the southern part and lowlands of the Serengeti plains in the northern and western parts. The majority of the inhabitants of the district are Maasai pastoralists. The district is administratively divided into 3 divisions, 14 wards and 40 villages. The district population is estimated at 136,135 of which 27,227 are children under the age of 5 years. Ngorongoro district has a total of 18 health facilities, comprised of 2 hospitals and 16 dispensaries. The majority of the inhabitants of the districts are pastoralists. The study involved four villages situated within the NCAA and lying at altitudes above $1700 \mathrm{~m}$. The villages were Osinon (1720m), Olbalbal (1780m) Endulen (1800m) and Oloirobi $(2100 \mathrm{~m})$.

\section{Retrospective morbidity and mortality malaria data} Malaria morbidity and mortality data were taken from health facilities, which provide health services for the study villages. The facilities included NCAA dispensary, Endulen Mission Hospital and Olbalbal Health Centre. The data were collected using the Health Management Information System (HMIS) Book 2/27(A-B) covering a period from 1991-2002.

\section{Malariometric survey}

A detailed malariometric screening was carried out to determine prevalence of malaria parasites, enlargement of spleen (splenomegaly), and haemoglobin levels. Thick and thin blood smears from finger-prick of approximately 150-200 individuals of both age groups were randomly selected and screened for malaria parasites in each of the selected villages. Smears were then stained with Giemsa stain and examined under microscope for malaria parasite species identification. Parasites were then enumerated against 200 leukocytes. Spleen rates were determined for both children and adults. However, haemoglobin measurements were taken from fifty individuals among pregnant women and children under 5 years.

\section{Entomological survey}

Mosquito collections were done using light trap and pyrethrum spray catch techniques. One room in each selected house was sprayed with pyrethrum during the morning hours. After 10 minutes, the mosquitoes knocked down on white sheets spread on room floor 
and horizontal surfaces were picked up and preserved in petri dishes lined with moist cotton wool and filter papers. Unfortunately, overnight human landing catches could not be done due to security reasons (the area being within the National Park). Data on parameters of interest like geographical location, housing construction, proximity to potential mosquito breeding sites and the presence of livestock were collected. Discussions with the communities were also made as to the possible source of mosquitoes to the village.

\section{Assessment of local knowledge, attitudes and perception on malaria}

Two communities were selected from each of the 4 study villages. Key informants were identified to assist the research team in recruiting participants and completing qualitative data collection, which included mainly an individual in-depth interview and direct observations. The interviews involved both heads of the household whenever they were available. The interviews used both structured and open-ended questionnaires. Observation method was employed to collect relevant data. Information related to illness classifications, perceived causes, usual resorts to care and relative perceptions of treatment options was collected. The assessment addressed normative attitudes toward malaria and malaria treatment options, an inventory of antimalarial drug sources and providers, and detailed illness narrative interviews. The survey also included questions about the recent use of antimalarial drugs by household members.

\section{Data analysis}

Morbidity data were collected and entered in a Microsoft Excel matrix that was prepared for the purpose. The monthly malaria cases for each year from 1996 to 2002 were analysed. Among the data for the five years, the largest and the $2^{\text {nd }}$ largest number of cases for each month were determined. A line graph was plotted for the $2^{\text {nd }}$ largest number of cases. This line graph served as a reference "normal malaria trend" comparing data for subsequent years. The line represented the upper normal limit of number of cases seen at the health facility. During the subsequent years, monthly malaria cases were plotted on the reference line graph. When the monthly number of cases exceeded a point on the original reference line graph or the upper normal limit, it indicated the beginning of an epidemic.

Malariometric data were entered into Epi-Info 6 and STATA for statistical analysis. Only data from Endulen Hospital were analysed and used to determine malaria epidemic threshold. Coding was done on data on knowledge, attitude and perception and was analysed using SPSS statistical package and STATA software programme.

\section{Results}

Malaria in Ngorongoro area showed a seasonal pattern with the highest peak in March-April and a small peak in October. When malaria cases reported at Endulen Hospital were plotted and compared with established epidemic alert and action lines, epidemics occurred in 1998, 2001 and 2002 (Figure 1). Between 1996 and 2002 malaria in Endulen Hospital accounted for 25$47 \%$ and $23-44 \%$ of all diagnoses in the $<5 y$ year and $\geq 5$ years, respectively.

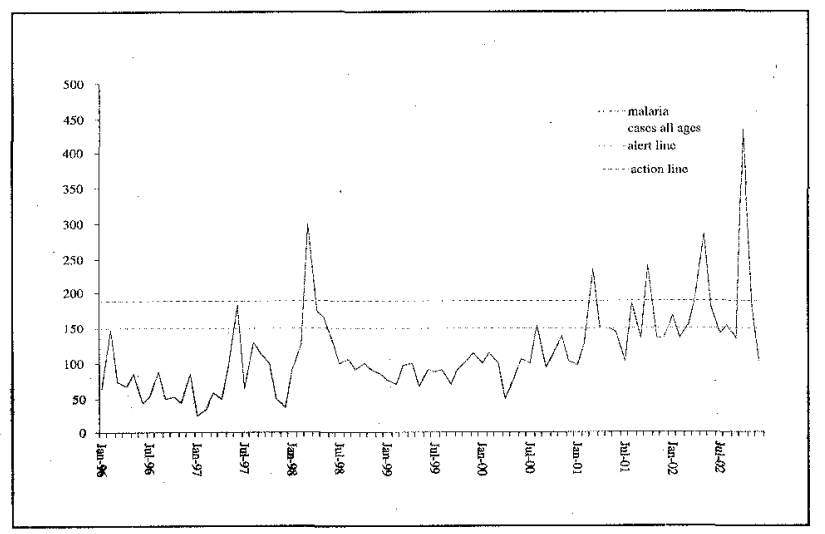

Figure 1: Pattern of malaria cases in all age groups January 1996-July 2002

A total of 739 individuals were examined in the four villages during the survey. Data collected suggest that malaria is spread across all study villages and Plasmodium falciparum was the only species identified in the area. There was a distinct pattern of parasite prevalence, with high malaria parasite rates among people living in relatively low altitudes than in the higher altitudes. In the order of increasing altitude the prevalence scores were as follows: Osinoni (25.9\%), Endulen (13.9\%), Olbalbal $(9.8 \%)$ and Oloirobi $(3.6 \%)$, indicating that there were almost a two-fold decrease of parasite prevalence between Osinoni village located at the lowest altitude and Oloirobi village located at the highest altitude (Table 1).

The intensity of infection was also reflected in the degree of malaria parasites in the blood of the screened individuals. In this regards residents of Osinoni were having a highest score (pooled parasite density) almost four-folds that of residents of Endulen and Olbalbal; and nineteen times that of Oloirobi. Parasite density distribution suggests that all age 
groups were potentially at risk of contracting malaria in all villages. In Osinoni, the highest burden was in the underfives, which accounted for $35 \%$ of all $<5$ years screened compared to individuals $\geq 5$ years old. However, male underfives were having more parasitaemia than their female counterparts. In Endulen the most affected individuals were those $\geq 5$ years old, indicating that this group was at the highest risk than the rest. In Olbalbal, the disease pattern was equally the same in all age groups with the exception of males $\geq 5$ years old, who had the highest (almost three-folds than the rest) parasitaemia rate. In Oloirobi all age groups were equally affected and had the least parasites density compared to residents of other villages. In this area ambient temperatures were $10-14^{\circ} \mathrm{C}$ during the survey.

The levels of haemoglobin $(\mathrm{Hb})$ among the $<5$ year old children were not significantly different between the villages. On average all $<5$ years old children in the study villages were having fairly good $\mathrm{Hb}$ levels ranging between 8.7 and $10.6 \mathrm{~g} / \mathrm{dl}$. However, when measurements were stratified by age groups and sex, scores for males and female $<5$ years old children ranged from 4.9 to 11.8 and 3.5 to 13.8 , in Osinoni and Olbalbal, respectively.

Very few individuals were having enlarged spleens in all study villages (Olbalbal $0.4 \%$, Oloirobi $0.7 \%$, Endulen $0.5 \%$, Osinoni $1.6 \%$ ); an observation suggesting that malaria could be a disease of recent
No malaria vector mosquitoes were collected in the study villages probably due to the cool and dry weather spell. Potential breeding sites were however, observed from a distance but could not be reached due to fear of wild animals. Olbalbal seasonal lake situated northwest of Olbalbal village was identified to one of the obvious potential breeding sites. In Oloirobi, swamps were mentioned to be the source of breeding ground for mosquitoes around the village. Lake Largarja at Ndutu, which is few kilometres northwest of Endulen, was also associated with the constant influx of anophelines in the village. Seasonal lakes and rivers southwest of the village were believed to be responsible for bringing malaria vectors in the village during peak transmission season. Nuisance mosquitoes (Culex quinquefasciatus) were abundant in Endulen reflecting the availability of long-term standing/polluted waters in the areas.

A total of 295 people were selected and interviewed using a convenient sampling design. The majority $(66.4 \%)$ of the respondents were aged between 25 and 44 years old. Over $94.9 \%$ of the respondents were married. In $98.0 \%$ of the households, men were the heads of the households. The majority $(55.4 \%)$ of the respondents were following traditional religions. Christians and Moslems accounted for $43.9 \%$ and $0.7 \%$, respectively. Moslems were only found in Osinoni. Endulen had the highest number of Christians. Most $(80.4 \%)$ of the inhabitants lived in

Table 1: Demographic characteristics of population in Ngorongoro area

\begin{tabular}{|c|c|c|c|c|}
\hline Variable & Village & Total & Male & Female \\
\hline \multirow[t]{4}{*}{ No. examined } & Olbalbal & 205 & 114 & 91 \\
\hline & Oloirobi & 139 & 55 & 84 \\
\hline & Endulen & 202 & 92 & 110 \\
\hline & Osinoni & 193 & 79 & 116 \\
\hline \multirow[t]{4}{*}{ Age range (years) } & Olbalbal & $0.75-59$ & $0.75-55$ & $1-59$ \\
\hline & Oloirobi & $0.25-79$ & $0.5-79$ & $0.25-76$ \\
\hline & Endulen & $2-68$ & $2-60$ & $2-68$ \\
\hline & Osinoni & $0.25-75$ & $1-75$ & $0.25-75$ \\
\hline \multirow[t]{4}{*}{ Mean age (years) } & Olbalbal & 10.98 & 7.24 & 11.40 \\
\hline & Oloirobi & 19.46 & 15.17 & 14.2 \\
\hline & Endulen & 17.4 & 10.28 & 11.2 \\
\hline & Osinoni & 20.61 & 15.49 & 14.84 \\
\hline
\end{tabular}

past. In Osinoni and Olbalbal all age groups were having splenomegaly compared to the rest of the villages, which had few splenomegaly scores in $\geq 5$ years old group only. This suggests that malaria is persistent and more serious in the former two villages than the rest. mud-grass thatched houses $($ Endulen $=50.6 \%$; Olbaba $=$ $87.0 \%$; Osinoni $=93.1 \%$; Oloirobi $=96.5 \%$ ) . Endulen village had the highest proportion $(42.5 \%)$ of houses with mud and corrugated iron roof construction. 
Table 2: Malaria parasite rate, splenomegaly and haemoglobin level by villages in Ngorongoro Area

\begin{tabular}{|c|c|c|c|c|}
\hline Variable & Osinoni & Olbabal & Endulen & Oloirobi \\
\hline Altitude (m asl) & 1720 & 1780 & 1800 & 2100 \\
\hline No examined & 193 & 205 & 202 & 139 \\
\hline Mean age (yr) & 20.6 & 11.0 & 17.4 & 19.5 \\
\hline$\%$ Parasite positive & 25.9 & 9.8 & 13.9 & 3.6 \\
\hline Mean parasite density/ul & 180 & 126 & 97.14 & 104 \\
\hline$\%$ Splenomegaly & 1.6 & 0.4 & 0.5 & 0.7 \\
\hline Mean $\mathrm{Hb}$ in males $(\mathrm{g} / \mathrm{d})$ & 8.8 & 10.1 & 9.4 & 9.6 \\
\hline Mean Hb in females $(\mathrm{g} / \mathrm{di})$ & 9.6 & 9.6 & 8.7 & 10.6 \\
\hline
\end{tabular}

The overall level of education was low, with only $44.6 \%$ of the respondents having had primary school education. The lowest literate rate was observed at Olbabal (29.8\%) and Oloirobi (27.9\%). Respondents with post-secondary school education were mostly found in Endulen.

The majority of the respondents were livestock herders $(94.2 \%)$, keeping mainly cattle, sheep and goats. There were however, fewer goats in Endulen than in other places. Respondents owning radios $(5.7 \%)$ were only found in Endulen.
The majority of the people especially in Olbabal and Endulen knew very well the symptoms of malaria. Most of the respondents mentioned headache, sequential chills, fever and sweating as the common symptoms of malaria. Of the 277 who responded to the question, $72.6 \%$ could mention the symptoms of malaria correctly. However, only $36.2 \%$ of the respondents in Oloirobi could correctly mention the symptoms of malaria. The majority $(59.4 \%)$ of the Oloirobi community associated malaria with fever

Table 3: Disease ranking among communities in Ngorongoro area

\begin{tabular}{llllll}
\hline Rank & Obalbal (\%) & Oloirobi (\%) & Endulen (\%) & Osinoni (\%) & Overall (\%) \\
\hline $\mathbf{1}$ & Malaria (90) & Fever (69) & Malaria (62) & Malaria (91) & Malaria (56) \\
$\mathbf{2}$ & Fever (48) & Pneumonia (26) & Typhoid (40) & Fever (61) & Fever (32) \\
$\mathbf{3}$ & Pneumonia (22) & Worms (21) & Brucellosis (23) & Pneumonia (42) & Pneumonia (23) \\
$\mathbf{4}$ & Tuberculosis (21) & Eye infection (17) & Tuberculosis (26) & Tuberculosis (30) & Tuberculosis (20) \\
$\mathbf{5}$ & Skin diseases (17) & Malaria (13) & Diarrhoea (21) & Eye infection (19) & Diarrhoea (15) \\
\hline
\end{tabular}

More than $50 \%$ of the respondents reported malaria as the major disease problem in the area. This was followed by fever, pneumonia, tuberculosis, and diarrhoea. There were quite some differences in disease ranking among the respondents in the four villages. Malaria was the most important disease in Obalbal, Endulen and Osinoni. Malaria was however, not considered as number one health problem in Oloirobi. In this village fever was considered as the most important health problem by $69 \%$ of the respondents (Table 3). Although malaria was classified as the most serious disease in Osinoni, more than $70 \%$ could hardly tell the name of any antimalarial drug.

Of the respondents, $76.2 \%$ said that at least a member of their household suffered from malaria during the past year. Only $39.5 \%$ of the respondents in Oloirobi had a malaria case during the reporting period. In $23.1 \%$ of the households, at least 2 people suffered from malaria during the past year. only. Joint pains were rarely associated with malaria in Olbabal and Endulen villages.

Most $(82.7 \%)$ of the respondents were obtaining antimalarial drugs from the nearest health facilities (hospital and dispensary). Kiosks and traditional healers were the major sources of antimalarial drugs for only $9.2 \%$ and $8.2 \%$ of the respondents, respectively. Traditional healers were however, the most important source of treatment for people in Olbabal (19.1\%) and Osinoni (17.6\%). Although the closest place people in Ngorongoro could obtain health care was the health facility, the burden on seeking treatment was pretty high since most $(59.7 \%)$ of them had to travel for more than four kilometres through a vast land to get medical attention.

Overall, knowledge on the cause of malaria as well as the transmission season was very high among the respondents. Eighty-seven percent of the respondents believed that malaria could affect anyone 
within the household and that the disease does not threaten children and women as a group. Mosquitoes were incriminated to bring about malaria in the four communities.

Most of the respondents mentioned that they sought treatment for malaria from a healthcare facility followed by folk medicine. When asked where most cases of malaria are treated, the majority $(87.4 \%)$ of the respondents would take the patients to health facilities. This was less common in Oloirobi (61.8\%) and more common in Endulen (98.8\%). Buying drugs was common in Osinoni $(10.3 \%)$ and rare in Oloirobi and Endulen. In Oloirobi, $38.2 \%$ of the people sought treatment from traditional healers.

Of 295 respondents, $62 \%$ knew names of antimalarial drugs. This was more common in Endulen $(85.1 \%)$ and less common in Osinoni (28.4\%). The majority $(84.7 \%)$ of the respondents said that most of cases of malaria occur during the rainy season and mosquitoes were mentioned by $85.7 \%$ of the respondents to be responsible for malaria transmission. Sulfadoxine pyrimethamine (SP) was known by the majority of the respondents $(52.7 \%)$, being more common in Endulen (77.3\%), less common in Osinon insecticide. The usefulness of insecticide treated nets was poorly known among the communities in Ngorongoro area.

Generally, a few people were found to keep drugs at home. This was most common (40.4\%) in Olbabal whereas none of the respondents from Endulen was keeping drugs at home. SP was the most common antimalarial drug kept at home by the majority of the respondents $(62.5 \%)$. This was observed more frequently at Osinoni. Chloroquine and amodiaquine were only found kept among the respondents in Olbabal. Interestingly, Cotrimoxazole was the most common drug kept by the majority of the respondents in Oloirobi.

Poverty, ignorance, ineffectiveness of drugs, poor sanitation, scarcity of water, culture and traditions were considered as the major factors contributing to the occurrence of communicable diseases such as malaria (Table 4). Ignorance was ranked high as a constraint in malaria control in Endulen and Osinoni. Culture and tradition were mentioned to be most important in Endulen whereas the majority of respondents from Olbabal and Osinoni mentioned poor sanitation.

Table 4: The most important contributing factor to the occurrence of diseases ( $\%$ of response), $N=293$

\begin{tabular}{lccccc}
\hline Factor & Oloirobi & Olbabal & Endulen & Osinoni & Total \\
\hline Poverty & 46.5 & 62.5 & 50.6 & 20.8 & 44.0 \\
Ignorance & 16.3 & 16.7 & 29.9 & 26.4 & 22.9 \\
Ineffectiveness of drugs & 27.9 & 0 & 0 & 0 & 8.2 \\
Poor sanitation & 2.3 & 12.5 & 2.3 & 18.1 & 7.9 \\
Scarcity of water & 0 & 2.1 & 0 & 19.4 & 5.1 \\
Traditions/culture & 2.3 & 0 & 10.3 & 5.6 & 5.1 \\
Others & 4.6 & 6.2 & 6.9 & 90.3 & 6.8 \\
\hline
\end{tabular}

(15\%). Quinine was well known in Osinoni and less so in Oloirobi. Chloroquine was still popular among the respondents of Osinoni $(20 \%)$ and Olbabal (21.3\%). Local herbs were commonly $(59.0 \%)$ used to treat malaria in Oloirobi.

Traditional healers were frequently contacted for treatment of malaria in Oloirobi by $59.3 \%$ of the people. Endukushi and Enekidong'o were mentioned as the most potent herbs used as antimalarials in the district. A significant number (22\%) of people in the district would do nothing to prevent themselves and their families from malaria. In addition, more than half of the respondents suggested that the best way to control malaria in the district would include the use antimalarial drugs and indoor house spraying with

\section{Discussion}

Our findings have shown that malaria is prevalent in all villages within Ngorongoro Conservation Area and occurs even at altitudes above $1,700 \mathrm{~m}$. In recent years, highland malaria has been reported in other districts of Tanzania (Mboera et al., 2002a,b; Kamugisha, 2005). The low prevalence of malaria (3.6-25.91\%) indicates that the area experiences an unstable malaria transmission and is prone to frequent malaria outbreaks. The variation in malaria prevalence may be due to many factors including biological, physical and socio-economic factors. Similar conditions are experienced in other highland areas $(>1000 \mathrm{~m})$ of Tanzania (Mboera, 2004). 
It is estimated that over $8 \%$ of the people in Tanzania live in malaria unstable areas prone to epidemics (Ministry of Health, 2000 unpublished report). The National Malaria Control Programme reports that 10 districts in Tanzania have reported at least one malaria epidemic since 1996. Currently, 16.7$20.8 \%$ of the districts in Tanzania are classified as malaria epidemic prone (Ministry of Health, 2000 unpublished).

In some villages, more male underfives had parasitaemia than their female counterparts. The reasons for this phenomenon could not be established. However, it is likely to be associated with the activities male children are involved in. In most pastoralist communities, boys are usually involved in herding livestock, moving their herds from place to place. This is likely to expose them more to mosquito bites, and hence higher malaria parasitaemia than their female counterparts.

Various studies have shown that malaria epidemics may be linked to environmental changes (such as increased mean rainfall and ambient temperatures) (Brown et al., 1998; Garay, 1998; Lindblade et al., 1999; Kilian et al., 1999; Molteni et al., 2002; Mboera et al., 2002a; Mboera et al., 2005) as well as socio-economic factors which include frequent movements, housing, and personal preventive measures (e.g. insecticide, mosquito nets, repellents, drugs). Although environmental changes that result from global and local processes are likely to have some effects on vectors of malaria, it is likely that the frequent movements among the pastoralists, who have low immunity against malaria, play a significant role in perpetuating malaria outbreaks. Frequent movements of people from the lowlands to highlands are likely to have introduced malaria parasites in previously malaria free areas of Ngorongoro district. Such movements are common among pastoralists, who spent most of the nights outdoors or indoors unprotected from mosquito bites. Frequent movements among the communities in Ngorongoro have been associated with tribal conflicts, epidemics of communicable diseases other than malaria, and search for greener pastures and water (S. Mkumbo, personal communication).

Despite frequent malaria epidemics in Ngorongoro district, ITN coverage is quite low. Most of those who own at least a mosquito net were found in Endulen, an area with the only hospital in the area. Recently, it has been reported in Iringa and Mpwapwa districts that more nets are owned by people living in villages with health facilities than in those without
(Mboera et al., 2002a). The perception that malaria could best be controlled by chemoprophylaxis and low knowledge on the benefits of the use of mosquito nets are also likely to have contributed to the low ITN coverage in the most of the villages in Ngorongoro area.

Community knowledge and perceptions play a significant role in the outcome of malaria in many places of Tanzania. Observations made in our study indicate that the nomadic community is relatively illiterate, has poor knowledge on malaria prevention techniques, consult traditional healers more often and have to travel long distance to seek treatment for malaria. These factors are likely to contribute to heavy tolls during malaria outbreaks. The number of householders whose first reaction is to consult a traditional healer for cases of fever is relatively high in some villages of Ngorongoro. The delay in seeking proper medical care has been observed in other malaria epidemic prone districts in Tanzania (Mboera \& Kitua, 2001; Mboera, 2004).

Striking information was observed in the utilization of folk medicine to treat malaria in Oloirobi village. Despite the fact that the village is close to a health facility, $60 \%$ of it inhabitants prefer traditional medicine. Traditional herbs such as Endukushi and Enekidong'o, were singled out as the most appropriate and effective antimalarial drugs. Further research is required to verify the effectiveness of Endukushi and Enekidong'o in fighting malaria and how it is ranked within the pattern of resorts in the village as illustrated by Kleinman (1980) given the disease severity, or Young's (1981) pattern, which incorporates assessment of cost and knowledge of the illness, and remedy efficacy. Probably the immediate factor that could be attributed to Oloirobi resident's preference to folk medicine is their strong belief and practice in traditional religion with almost ninety percent acknowledging so.

Already several studies have shown that communities in highland areas have poor knowledge on malaria disease and its transmission when compared to those in endemic lowland areas (Mboera et al., 2002b; Rumisha et al., 2003). This poor knowledge prompts such community to seek treatment late and most of the times after consulting traditional healers. Home treatment practices may also play a great role in delay in seeking care, and when it is during epidemics, loss of life may be experienced (Mboera, 2004). 
While the findings portray that people in Ngorongoro district possess a very good knowledge on what transmits malaria and possible ways to treat the disease, they have poor knowledge on malaria prevention and limited access to reliable health facility services. The fatality of malaria outbreaks worsens when access to effective treatment is limited. The distance problem is worsened by the fact that the villages are located within the Ngorongoro wildlife area, which is home to a number of wild and hostile animals such as lions, hyenas and buffaloes, and the fact that most cannot afford the cost of transport even when it is provided by the Conservation Authority.

The gap between knowledge and practice plays a crucial role in malaria mismanagement in Ngorongoro. Even though a handsome number of respondents mentioned chemoprophylaxis as the best way to prevent and control it, but for others, their choice of drugs leaves a lot to be desired. Almost a half of those reported to hoard drugs at home mentioned antipyretics such as paracetamol and aspirin as appropriate drugs for treating malaria (data not shown). Similar findings have been reported by Mutalemwa et al. (2003) in a study conducted in Tanga district in north-east Tanzania.

Only a few people in Ngorongoro are aware that mosquito nets can be used for personal protection against malaria mosquitoes. This knowledge gap requires a rapid response in order to prevent new cases. However, given the complaints of low socio-economic level among the population, appropriate cost effective preventive mechanisms should be put in place. This may involve the use of discount voucher system to purchase treated nets which has in a way proven useful in some areas in Tanzania (Mushi et al. 2003).

Frequent epidemics of communicable disease have been associated to poor district epidemic preparedness (Mboera et al., 2005). It has been observed that, in many districts, outbreaks are not detected, not confirmed and not reported timely. To minimize heavy death toil and sufferings during malaria epidemic, an early warning system and epidemic preparedness plan must be developed in each facility in the district. The development of a warning system at facility and district levels is an important tool to predict outbreaks and hence, save many lives.
To do this, districts need to have reliable databases and carry out routine analysis of epidemiological surveillance data. There is need for Ngorongoro district in collaboration with the Ngorongoro Conservation Area Authority, therefore to develop health database that can be used as a source of information for spatial and temporal distribution of malaria. Such a database has been developed for each health facilities in Mpwapwa (Mboera et al., 2005) and Dodoma Rural districts.

Since communities in Ngorongoro have poor knowledge on malaria prevention and control, health education and promotion through community participation will be an important aspect in malaria interventions. There is a great window of opportunity therefore for the district to initiate a health education and promotion programme for the communities in the area. Given the inadequate knowledge on malaria control and lack of an early warning system in the district, malaria outbreaks will continue to occur in the Ngorongoro area. This is likely to happen if immediate and strategic course of action is not taken to impart health-related education on malaria and other life threatening diseases. The health education should focus on both appropriate preventive and curative mechanisms that include correct chemotherapy, and advocating the use of insecticide treated mosquito nets.

\section{Acknowledgements}

The authors want to particularly thank Mr. Eric Mfinanga, Peter Sami, Abdallah Telaki and Martin Zuakuu for their excellent technical and field assistance. Dr. S. Mkumbo of the Ngorongoro Conservation Area Authority is thanked for his logistical support and comments in the earlier version of the manuscript. This study received financial assistance from the Ngorongoro Crater Conservation Authority.

\section{References}

Alilio, M.S., Njunwa, K.S. \& Msuya, F.H. (1995) The changing pattern of malaria mortality and morbidity in Ngorongoro District, Arusha: Social economic assessment. Annual Report of the National Institute for Medical Research 14, 31 .

Brown, V., Isaak, M.A., Rossi, M., Barboza, P. \& Paugam, A. (1998) Epidemic of malaria in northeastern Kenya. The Lancet 352, 1356-1357. 
Clyde, D.F (1967) Malaria in Tanzania. London, Oxford University Press.

Ellman, R., Maxwell, C., Finch, R. \& Shayo, D. (1998) Malaria and anaemia at different altitudes in the Muheza District of Tanzania: Childhood morbidity in relation to level of exposure to infection. Annals of Tropical Medicine and Parasitology 92, 741-753.

Garay, J. (1998) Epidemiological Survey and Situation Analysis: Malaria Epidemic in Nshamba Division, Muleba District, Tanzania. MSF Spain. 47 pp.

Kamugisha, M.L. (2005) The effect of endemicity on the sensitivity and specificity of malaria case definition and attributable fraction in northern Tanzania. Tanzania Health Research Bulletin 7, 1-6.

Kilian, A., Langi, P., Talisuna, A. \& Kabagambe, G. (1999) Rainfall pattern, El Nino and malaria in Uganda. Transactions of the Royal Society of Tropical Medicine and Hygiene 93, 22-23.

Kleinman, A. (1980) Patients and Healers in the Context of Culture. Berkeley; University of California, USA.

Lindblade, K.A., Walker, E.D., Onapa, A.W., Katungu, J. \& Wilson, M.L. (1999) Highland malaria in Uganda: prospective analysis of an epidemic associated with El Nino. Transactions of the Royal Society of Tropical Medicine and Hygiene 93, 480-487.

Mboera, L.E.G. (2004) Environmental and socioeconomic determinants of malaria epidemics in the highlands of Tanzania. Tanzania Health Research Bulletin 6, 11-17.

Mboera, L.E.G., Kamugisha, M.L., Malima, R.C., Mushi, A.K., Msuya, F.H., Masawe, T. \& Kitua, A.Y. (2002b) Malaria prevalence and health seeking behaviour among communities of the lowlands and highlands of Gonja, Same District, north-eastern Tanzania. Tanzania Health Research Bulletin 4, 47-53.

Mboera, L.E.G., Kisinza, W.N., Kamugisha, M.L., Rumisha, S.F., Chuwa, G.J., Kitua, A.Y., Molteni, F. \& Majori, G. (2002a) The impact of altitude and access to health services on malaria prevalence among schoolchildren in Iringa Rural District, Tanzania. Public Health Situation in Tanzania: Re-packaging Knowledge for Health Promotion. In: Proceedings of the 20th Annual Scientific Conference of the Tanzania Public Health Association, November 19-23, 2002, Mbeya, Tanzania. Pp. 66-71.

Mboera, L.E.G. \& Kitua, A.Y. (2001) Malaria epidemics in Tanzania; An overview. African Journal of Health Sciences 8, 14-18.

Mboera, L.E.G., Molteni, F., Nyange, A. \& Thomas, E.G. (2005) Using retrospective epidemiological data to determine malaria epidemic prone areas and development of an epidemic early warning system in Mpwapwa District, Central Tanzania. Tanzania Health Research Bulletin 7, 73-78.

Mushi, A.K., Armstrong-Schellenberg, J.R.M., Mponda, H. \& Lengeler, C. (2003) Targeted subsidy for malaria control using discount voucher system in Tanzania. Health Policy and Planning 18, 163-171.

Mutalemwa P.P., Mboera, L.E.G. \& Mittelmark, (2003) Living with Malaria in Tanzania: An Insight from a Rural Community of Tanga District. Tanzania Health Research Bulletin 5, 13-18.

Rumisha, S.F., Mboera, L.E.G., Kisinza, W.N., Chuwa, G.J., Kitua, A.Y. \& Molteni, F. (2003) Community perceptions of malaria and its management in Iringa District, south-western Tanzania. Tanzania Health Research Bulletin 5, 41-47.

WHO (1997) Health and Environment in Sustainable Development. Five years after the Earth Summit. World Health Organization, Geneva. WHO/EHG/ 97.8.

WHO (2001) Malaria Early Warning Systems. Concepts, Indicators and Partners: A Framework for Field Research in Africa. WHO/CDS/RBM/ 2001.32, Geneva.

Young, J.C. (1981) Medical Choice in a Mexican Village. New Brunswick: Rutgers University Press. 